\title{
IL CONTRIBUTO DEL POLITECNICO ALLO SVILUPPO DELL'INFORMATICA
}

\author{
CARLO GHEZZI (*)
}

SuNTO. - L'ingegneria informatica nasce a livello internazionale nel dopoguerra e si afferma come una delle discipline di maggior impatto economico, industriale e sociale nell'ultimo decennio del secolo scorso. La sua nascita al Politecnico può farsi risalire all'inizio della seconda metà del secolo scorso, quando nel 1954 il Prof. Luigi Dadda portò in Italia dagli Stati Uniti il primo computer e iniziò le prime ricerche e sperimentazioni. Da allora l'informatica ha avuto uno sviluppo straordinario, che l'ha portata a costituire oggi il motore della società contemporanea, non a caso definita la società dell'informazione. In questo lavoro si traccia un'analisi storica dello sviluppo dell'informatica al Politecnico e del contributo apportato sia a livello nazionale che internazionale nella formazione e nella ricerca.

$$
* * *
$$

ABSTRACT. - The history of Computer Science and Engineering (Informatics) began internationally after the Second World War. In the last decade of the twentieth century it bacame one of the disciplines with highest impact on economy, industry, and society. The development of Informatics at Politecnico started when the first computer was brought to Italy from the USA by Prof. Luigi Dadda and the first experiments and investigations were launched. Since then Informatics has been continuously growing until today it became the engine of modern society, often called the Information Society. This paper reports on the main developments of Informatics at Politecnico and the main contributions achieved nationally and internationally in education and research.

(*) Istituto Lombardo Accademia di Scienze e Lettere, Politecnico di Milano, Italia. E-mail: carlo.ghezzi@polimi.it 


\section{NASCITA DELL'INFORMATICA MODERNA}

Linformatica ha radici antiche, che affondano nei primi tentativi di automatizzare il trattamento dei dati e delle operazioni aritmetiche da parte delle civiltà antiche. La nascita moderna dell'informatica risale però alla seconda metà del XX-esimo secolo. Limpulso dato dai governi alla ricerca durante seconda guerra mondiale, volta ad esempio ad automatizzare il calcolo delle traiettorie balistiche per il puntamento dell'artiglieria e lo sviluppo di algoritmi per violare i messaggi cifrati inviati dai nemici, ha portato alla nascita dei primi computer. Da qui, nel giro di pochi decenni, l'informatica ha segnato l'inizio di un passaggio epocale verso ciò che oggi chiamiamo comunemente la società dell' informazione.

Con la costruzione dei primi computer, l'informatica viene inizialmente a identificarsi con un mezzo fisico: il computer, e cioè lo strumento che la rendeva visibile. I primi computer, chiamati mainframe, sono oggi irriconoscibili come tali anche da parte di chi fa un uso quotidiano di dispositivi di elaborazione. La Fig. 1 ci mostra uno dei più famosi progenitori della famiglia dei computer: l'ENIAC4, sviluppato alla fine della seconda guerra mondiale all'Università della Pennsylvania. È interessante ricordare oggi alcuni dati relativi a questo computer: per la sua grande dimensione, era richiesto uno spazio di circa 170 metri quadri; il peso era di 30 tonnellate. Produceva una enorme quantità di calore, e quindi richiedeva un sistema di raffreddamento per controllarne la temperatura. Il computer assorbiva tanta energia elettrica che, alla sua prima messa in funzione, causò un blackout in un quartiere di Filadelfia. Il suo costo fu di 480 mila dollari di quell'epoca.

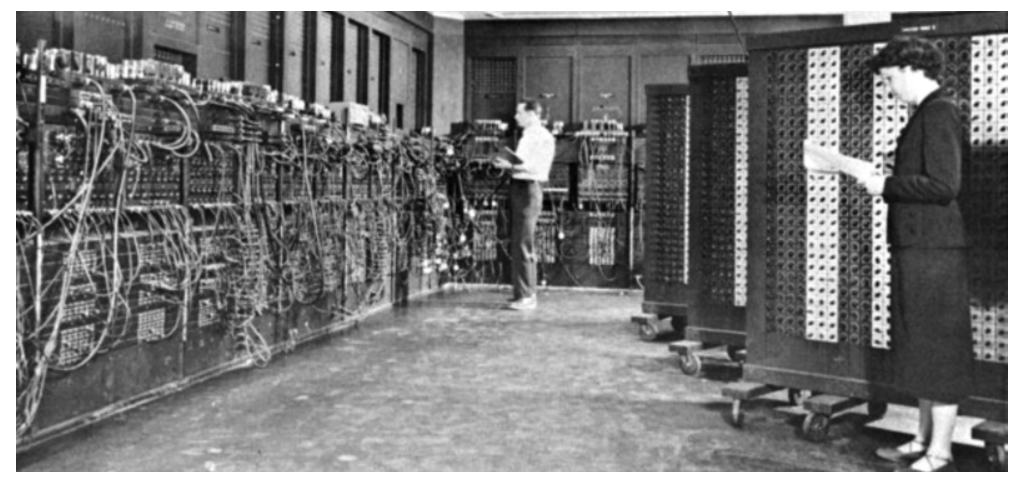

Fig. 1. ENIAC4. 
Malgrado questi dati impressionanti in termini di costo, dimensione e consumo energetico, questo computer ci fa sorridere se lo confrontiamo con i sistemi di elaborazione di oggi: non riusciva a trattare più di 20 numeri per volta contenuti in memoria e aveva una potenza neppure lontanamente paragonabile non solo a un comune personal computer (era 10mila volte più lento), ma anche ai telefoni cellulari che ci assistono nella vita quotidiana.

I primi computer erano estremamente fragili da un punto di vista tecnologico e richiedevano continui interventi di riparazione, che (come illustrato in Fig. 2) richiedeva spesso la sostituzione dei singoli dispositivi della macchina. La programmazione di questi computer era un'operazione lunga e complessa, che richiedeva di intervenire addirittura al livello di connettori fisici, come mostrato in Fig. 3.

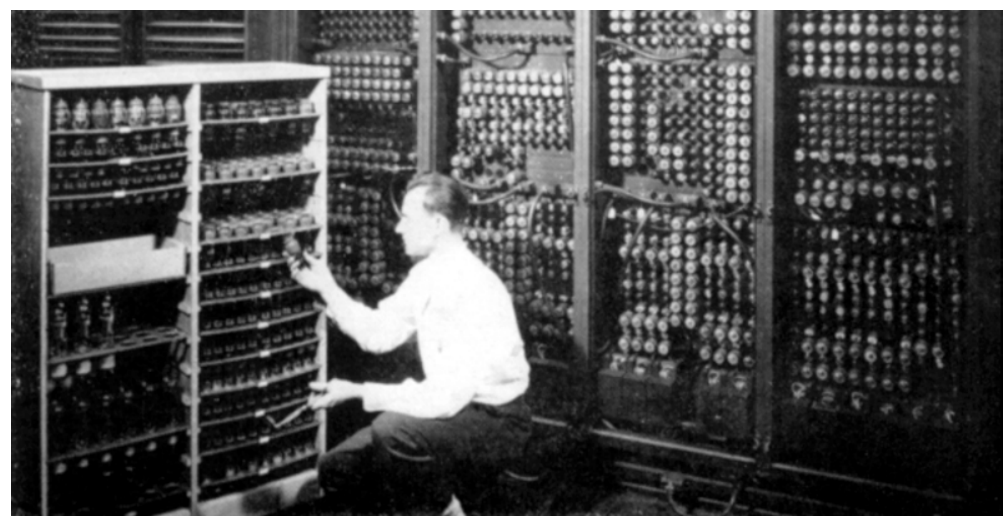

Fig. 2. Continui interventi di riparazione dell'bardware.

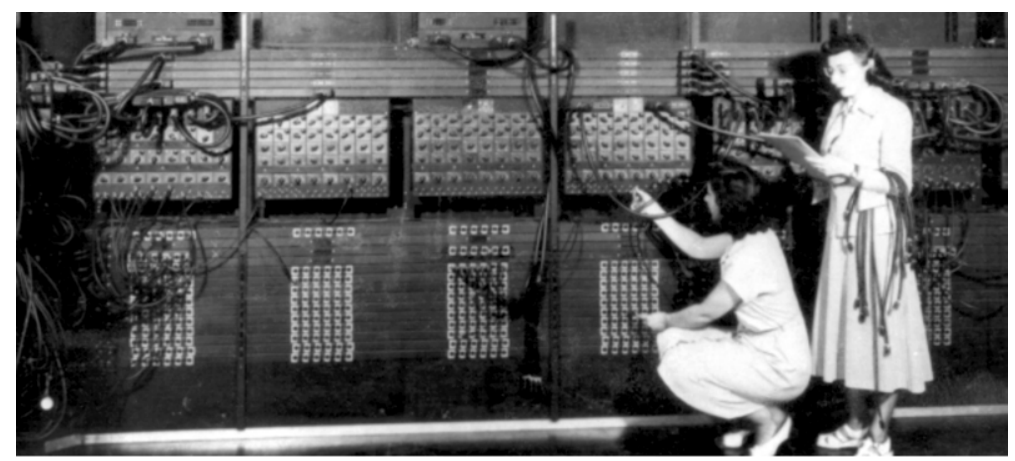

Fig. 3. Le prime forme di "programmazione". 


\section{NASCITA ED EVOLUZIONE DELL'INFORMATICA IN ITALIA E}

\section{Al PoliteCnico}

Nel 1954 inizia la storia dell'informatica in Italia (e al Politecnico). Il primo computer, mostrato in Fig. 4 da una foto d'epoca, arriva in Italia per merito della lungimiranza del Politecnico di Milano (e del suo rettore di allora, il Prof. Cassinis). Sfruttando un finanziamento ottenuto attraverso il piano Marshall, il rettore incarica dell'avventura un suo brillante giovane ricercatore (un trentenne!): il Prof. Luigi Dadda.

Il computer, denominato CRC 102A, del costo di 120.000 dollari, era prodotto a San Diego dalla Computer Research Corporation. Trattandosi però di un prodotto estremamente innovativo, era previsto che chi dovesse poi utilizzare la macchina dovesse partecipare anche alla sua costruzione, in quanto l'elaboratore, dopo la consegna, non sarebbe più stato assistito dal produttore. Il Professor Dadda dunque partecipò alla costruzione di questo primo computer. La macchina ultimata venne poi caricata su una vecchia nave da trasporto nel centro di una stiva piena di balle di cotone che avevano lo scopo di proteggere le valvole termoioniche che ne costituivano le principali unità dalle pericolosissime vibrazioni.
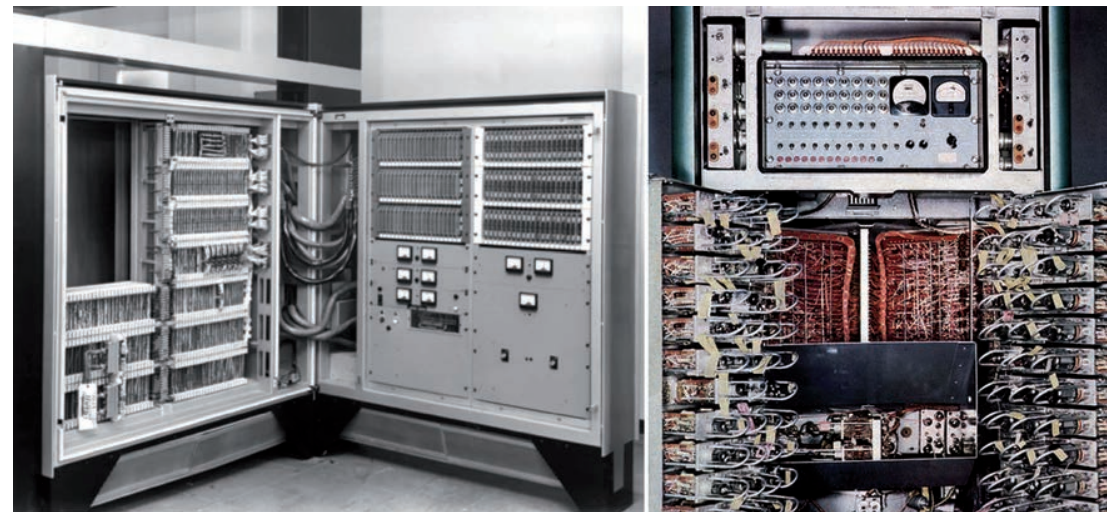

Fig. 4. CRC 102A, il primo calcolatore in Italia.

Vale la pena riflettere su questa avventura scientifica che fu il frutto di una straordinaria e coraggiosa lungimiranza. La prima lezione che si può trarre è che per uscire dalla crisi terribile in cui il Paese era sprofondato con la guerra e per affrontare il problema della ricostruzio- 
ne, il Politecnico decise di utilizzare il finanziamento ottenuto investendo per aprire una nuova area di ricerca, e non, ad esempio, per costruire edifici o impianti tradizionali. Questa lezione ci insegna che la ricerca, allora come oggi, può rappresentare il più efficace investimento per il futuro. Possiamo anche trarre una seconda importante lezione: in un momento di grande difficoltà, ma al tempo stesso di grande fermento, per mettere le basi alla ricostruzione del paese, si decise di investire su un giovane ricercatore: un giovane capace di mettersi in gioco per aprire un settore nuovo e ad alto rischio, visto che non era certo prevedibile all'epoca lo sviluppo impressionante che il settore avrebbe avuto successivamente. La coraggiosa lungimiranza che il Politecnico dimostrò allora è oggi ancora attuale e va recuperata urgentemente per riportare dinamismo ed entusiasmo che rimetta in moto la crescita nella nostra società.

Vale poi la pena ricordare che quell'investimento fu largamente ripagato: quel giovane trentenne, il Prof. Luigi Dadda, diventerà protagonista della storia dell'informatica nazionale, oltrechè scienziato di grande livello internazionale. Diventerà anche protagonista della storia del Politecnico di Milano, di cui sarà rettore dal 1972 al 1984.

Il computer CRC 102A venne installato nel settembre 1954 al Politecnico nel sottoscala dell'aula 2 SUD e risultò essere il primo computer funzionante in Italia e nell'Europa continentale. Negli anni successivi si svilupparono al Politecnico numerose attività, sul piano della conduzione tecnica del computer, della sua utilizzazione per applicazioni scientifiche e industriali, della ricerca nell'ambito dell'hardware e della formazione di docenti e studenti.

Con il primo computer nacque e progressivamente si rafforzò il legame inscindibile tra ricerca, formazione e trasferimento delle conoscenze e della tecnologia verso il mondo esterno nel settore dell'informatica, seguendo il modello da sempre interpretato con successo dal Politecnico in altri settori dell'ingegneria. Contemporaneamente alle iniziative del Politecnico, altre iniziative pionieristiche venivano avviate a Pisa, sia in ambito universitario che in ambito industriale da parte della società Olivetti. A Pisa nacque anche nel 1969 il primo corso di laurea a livello nazionale, dal titolo "Scienza dell'Informazione". A costituire il corpo docente di questo corso vennero chiamati numerosi giovani laureati al Politecnico di Milano, che si trasferirono a Pisa insieme al Prof. Antonio Grasselli, già assistente ordinario di Elettrotecnica e professore incaricato di Commutazione e Calcolatrici Elettroniche (1962-67) al Politecnico. 
Per quanto riguarda l'evoluzione del settore informatico al Politecnico, con riferimento all'organizzazione della ricerca, si ricordano le seguenti tappe fondamentali:

- 1955: il Prof. Ercole Bottani, già direttore dell'Istituto di Elettrotecnica Generale, diviene direttore anche dell'Istituto di Comunicazioni Elettriche;

- 1963: si fondono i due istituti di Elettrotecnica Generale e di Comunicazioni Elettriche nell'Istituto di Elettrotecnica ed Elettronica;

- 1982: l'Istituto di Elettrotecnica ed Elettronica diviene Dipartimento di Elettronica;

- 1992: il Dipartimento di Elettronica diventa Dipartimento di Elettronica e Informazione;

- 2013: il Dipartimento di Elettronica e Informazione diventa Dipartimento di Elettronica, Informazione e Bioingegneria.

Questa evoluzione organizzativa mette in evidenza come inizialmente l'Informatica al Politecnico venisse vista come parte dell'Elettronica, enfatizzando in tal senso l'aspetto costruttivo delle macchine. Successivamente, con l'aggiunta del termine "Informazione", l'informatica trova una visibilità autonoma nell'organizzazione dipartimentale.

La struttura formativa andò ad evolvere in parallelo con gli sviluppi della ricerca e con i continui sviluppi tecnologici, attraverso due momenti fondamentali:

- 1960: dal Corso di Laurea in Ingegneria Elettrotecnica nasce il corso di laurea in Ingegneria Elettronica. Il corso è inizialmente a numero chiuso e si differenzia dal corso di origine per gli insegnamenti del IV e del $\mathrm{V}$ anno. All'interno del corso di laurea, si collocano insegnamenti specifici di di Calcolatori Elettronici e di Programmazione;

- 1990: dal corso di laurea in Ingegneria Elettronica nasce il corso di laurea in Ingegneria Informatica.

Anche la formazione in Informatica nasce pertanto dall'Elettronica, coerentemente con l'enfasi iniziale sugli aspetti costruttivi (hardware) dei dispositivi di elaborazione. Già negli anni 70 , tuttavia, insegnamenti sui temi del software (ad esempio, relativi al linguaggi di programmazione, $\mathrm{i}$ compilatori, l'ingegneria del software, i sistemi operativi) si affiancarono ai corsi esistenti per arricchire la formazione dell'Ingegnere Informatico in una direzione nella quale il mondo si stava avviando in maniera impetuosa. Mentre agli inizi dello sviluppo dell'informatica le maggiori difficoltà tecniche e i maggiori costi di sviluppo risiedevano negli aspetti hardware, la situazione si è infatti completamente ribaltata con il passare 
del tempo, rendendo il software l'aspetto più critico all'interno dei sistemi informatici. Al tempo stesso, il software ha permesso lo sviluppo di applicazioni che hanno rivoluzionato la società contemporanea a partire dalla fine del secolo scorso.

\section{LA SITUAZIONE ATTUALE E IL FUTURO}

L'informatica si è oggi affermata come una delle aree più rilevanti della presenza nazionale ed internazionale del Politecnico di Milano, sia per quanto attiene la formazione che per quanto riguarda la ricerca. Tre sono gli aspetti su cui si è focalizzata l'attenzione dei ricercatori a livello internazionale, e quindi anche dei docenti del Politecnico:

- l'hardware,

- il software, che consente di dare vita, intelligenza, e valore aggiunto ai dispositivi di elaborazione,

- i sistemi e le applicazioni.

Per quanto riguarda l'hardware, in Fig. 5 si mostra come la ricerca abbia portato la tecnologia verso una continua riduzione di dimensioni (accompagnata da una riduzione dei costi e da un aumento delle prestazioni) delle unità di elaborazione. Al tempo stesso, la proliferazione di dispositivi ha portato progressivamente verso la situazione attuale di informatica distribuita e pervasiva che caratterizza il mondo nel quale oggi viviamo.

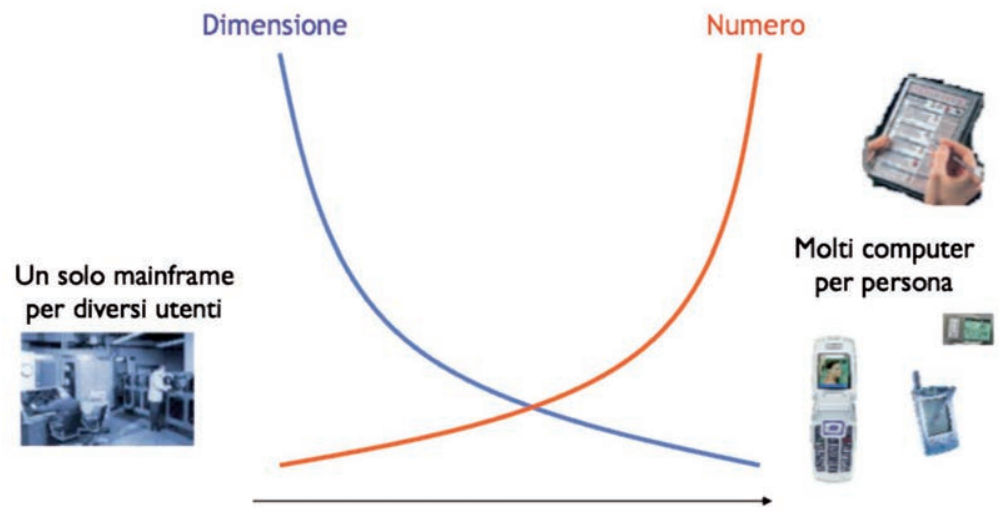

Fig. 5. Evoluzione della tecnologia: riduzione delle dimensioni e diffusione dei dispositivi di elaborazione. 
Per quanto riguarda il software, questo si presentò come un'area scientifico-tecnologica del tutto nuova rispetto alla tradizione di una scuola politecnica. Merito del Politecnico di Milano è stato il fatto di comprendere l'importanza del settore e di investire in esso. Per la prima volta nella storia della scienza e dell'ingegneria ci si è trovati infatti ad affrontare il problema di comprendere la natura di un materiale costruttivo "immateriale", che non ha fisicità, che non si riesce a visualizzare e che non si sapeva come progettare o misurare. Ci si è posto il problema di individuare i principi e i metodi che un ingegnere deve utilizzare per costruire con il software le nuove macchine astratte, diverse dalle tradizionali macchine fisiche, terreno delle ingegnerie tradizionali. Problema che gli ingegneri di aree storicamente più consolidate (come la meccanica, la chimica o le costruzioni civili) si sono posti attraverso decenni o addirittura secoli, e che hanno in larga misura risolto da tempo. Il problema è diventato cruciale per il software in quanto una cattiva progettazione può generare malfunzionamenti durante l'utilizzo che possono avere effetti gravi o addirittura disastrosi. Si pensi ad esempio a errori nel software che governa una transazione finanziaria o in quello che gestisce il volo degli aerei o in quello che controlla la guida delle automobili (per esempio, l'ABS) o ancora in quello che controlla le radiazioni emesse da un apparecchio per la radioterapia.

Per quanto riguarda la focalizzazione sui sistemi e le applicazioni, come abbiamo osservato, l'informatica è diventata sempre più distribuita e pervasiva, attraverso la diffusione e l'interconnessione dei dispositivi di elaborazione. Sempre più viviamo in un mondo in cui dispositivi di elaborazione sono sono inseriti nel mondo fisico per interagire con esso o per raccogliere dati che vengono poi convogliati in una rete informativa di scala planetaria che consente di superare ogni barriera di distanza in spazio e tempo. L'informatica consente non solo l'interconnessione degli elaboratori, ma attraverso ciò genera un nuovo spazio "ciber-fisico" in cui le persone operano, lavorano, vengono educate, si divertono, interagiscono tra di loro e con il mondo fisico.

Per far fronte alla domanda di ricerca e formazione in questi ambiti, il Politecnico ha fortemente investito in risorse intellettuali, fino ad arrivare alla situazione attuale, nella quale dispone di personale di ruolo così suddiviso (alla data di inizio 2014):

- 17 Professori Ordinari

- 27 Professori Associati

- 30 Ricercatori 
Accanto al personale di ruolo, numerosi studenti di dottorato e assegnisti di ricerca contribuiscono a creare un terreno estremamente fertile per la ricerca scientifica e la formazione avanzata.

La ricerca in informatica è estremamente attiva nell'ambito dei progetti di ricerca nazionali ed internazionali, in particolare nei progetti di ricerca finanziati dall'Unione Europea attraverso i programmi quadro. Due prestigiosi “Advanced Grant” dell'European Research Council sono stati conferiti a ricercatori in Informatica del Politecnico.

Per quanto riguarda la formazione, vengono oggi immatricolati oltre 500 studenti alla laurea di I livello e oltre 250 alla laurea di II livello, con un'offerta didattica complessiva di circa 200 corsi.

\section{CONCLUSIONI}

Il Politecnico di Milano ha dimostrato in passato di essere capace di anticipare l'evoluzione dell'informatica, aprendo nuovi ambiti di ricerca e trasferendo gli sviluppi della ricerca e della tecnologia nella formazione degli ingegneri e in progetti sviluppati in collaborazione con il mondo industriale. La nuova era dell'informatica diffusa e pervasiva apre un'orizzonte vastissimo di potenziali nuovi sviluppi. La ricerca, e con essa la formazione, dovranno sempre più focalizzarsi, oltre che sui temi "interni" alla disciplina, anche su temi "interdisciplinari". La grossa sfida "interna" è quella di arrivare a costruire sistemi altamente affidabili (e di affidabilità "garantita") che siano al tempo stesso in grado di evolvere in maniera il più possibile autonoma. Le sfide dell'interdisciplinarità sono poi numerosissime e molte sono oggi imprevedibili. L'obiettivo ultimo è il miglioramento della qualità della vita e la sostenibilità del suo sviluppo.

A titolo di esempio, è innegabile che il mondo occidentale si sta avviando a realizzare una società sempre più popolata da anziani. Diventa pertanto fondamentale capire come supportare l'autonomia di vita delle persone, attraverso una riprogettazione completa dello spazio in cui queste vivono. Per affrontare questo problema, si devono incontrare medici, sociologi, urbanisti, ingegneri edili, economisti, ingegneri gestionali, designers, trasportisti e (certo non ultimi) informatici. In questa integrazione, l'informatica gioca infatti un ruolo fondamentale, in quanto fornisce al sistema sia il tessuto nervoso che lo interconnette che l'intelligenza che lo fa funzionare. 
Il Politecnico dovrà dimostrarsi capace di interpretare questa domanda di progettualità del futuro, come ha dimostrato di saper fare nei 150 anni della sua storia passata. Un piccolo esempio di una recente riorganizzazione dipartimentale ci fa capire che esiste la volontà di muoversi in questa direzione. Il Dipartimento di Elettronica e Informazione è recentemente diventato Dipartimento di Elettronica, Informazione e Bioingegneria, aggregando al proprio interno l'ingegneria bio-medica. Questo può essere un meccanismo efficace per favorire una progettualità interdisciplinare in un settore di rilevanza sociale primaria, come quello della salute. Altri meccanismi dovranno essere individuati per altri ambiti interdisciplinari e altre iniziative dovranno essere sviluppate perchè il Politecnico continui a svolgere il suo ruolo chiave nella ricerca, la formazione e l'innovazione del paese. 